\title{
A hard road in psychiatric genetics: schizophrenia and DPYSL2
}

\author{
Makoto Arai and Masanari Itokawa \\ Journal of Human Genetics (2010) 55, 397-399; doi:10.1038/jhg.2010.66; published online 3 June 2010
}

$\mathrm{S}^{\mathrm{s}}$ chizophrenia is thought to be a hetero$\checkmark$ geneous syndrome with a prevalence of approximately $1 \%$ worldwide. Meta-analyses of twin studies estimated the heritability of schizophrenia as approximately $80 \%$, suggesting that a genetic study is an effective way to reveal the etiology of this disorder. However, genetic studies of schizophrenia have generally been disappointing, as the data obtained often cannot be reproduced. Reporting in this issue, Koide et al. ${ }^{11}$ investigated the DPYSL2 gene using the $\mathrm{MACH}$ program, which is one of the software packages that is popular for genotype imputation methods $(\mathrm{MACH}$, IMPUTE, fastPHASE, PLINK, and Beagle). Such information is currently available as a standard procedure in genetic association studies without genotyping/sequencing. ${ }^{1}$

Koide et al. chose the gene as a candidate involved in vulnerability for schizophrenia because it is coded on chromosome $8 \mathrm{p} 21$, which is reported as a linkage locus for the disorder. Human dihydropyrimidinase-like 2 (DPYSL2), also known as collapsin response mediator protein 2 (CRMP2), was originally identified from a fetal brain complementary DNA library (Online Mendelian Inheritance in Man (OMIM) 602463). DPYSL2, ubiquitously expressed in the fetal and neonatal brain, is involved in the developmental processes of the nervous system, such as cell differentiation, nervous system development, regulation of axon extension and signal transduction (http://www.genecards.org/cgi-bin/ carddisp.pl?gene $=$ DPYSL2\&search $=$ DPYSL2\& $\mathrm{rf}=/$ home/genecards/current/website/carddisp. pl\&go_cc=7\#go_cc). This was the second

Dr M Arai and Dr M Itokawa are at the Project for Schizophrenia and Affective Disorders Research, Tokyo Institute of Psychiatry, Schizophrenia Research Team, 2-1-8 Kamikitazawa, Setagaya, Tokyo 156-8585, Japan. E-mail: aria-mk@igakuken.or.jp reason why the authors chose DPYSL2 as a target for their current study, as intense research into schizophrenia clearly points to the involvement of a significant neurodevelopmental component.

The pathway of axon guidance (axon repulsion (Kyoto Encyclopedia of Genes and Genomes (KEGG) pathway; http://www. genome.jp/dbget-bin/www_bget?pathway+ hsa04360) is a key stage in the formation of the neuronal network. Several molecules, such as c-Fes, ${ }^{2}$ CDK5 (cyclin-dependent kinase 5$)^{3}$ and ROCK2 (Rho-associated, coiled-coil containing protein kinase 2), ${ }^{4}$ have been shown to phosphorylate DPYSL2 (denoted as CRMP2 in Figure 1), and DPYSL2 binds to tubulin heterodimers to promote microtubule assembly, which is important for axon outgrowth and branching. ${ }^{5-7}$ Ephrin-A receptors/Ephexin/Rho A cascade is also an important regulator of cytoskeleton rearrangement (Figure 1).

Several studies have reported the association between DPYSL2 and psychiatric disorders, including schizophrenia, ${ }^{8-10}$ bipolar disorders, neuroblastoma, epilepsy and Down syndrome. Nakata et al. ${ }^{9}$ reported that the ${ }^{\star} 2236 \mathrm{C}$ allele in the $3^{\prime}$ untranslated region of DPYSL2 was associated with schizophrenia, especially paranoid type (odds ratio 0.49 ), and they described the possible involvement of the nucleotide change in the regulation of mRNA stability or translational efficiency. However, replication studies in some populations have been inconsistent (SZGene; http://www.schizophreniaforum.org/ res/sczgene/default.asp).

Our preliminary data regarding DPYSL2 (CRMP2) mRNA levels in schizophrenia showed that the expression in lymphoblastoid cells derived from patients with schizophrenia and control subjects is not significantly altered. However, our data suggested that DPYSL2 is still a promising candidate, as Ephrin-A receptors, ROCK2 and CDK5 might be implicated in the regulation of DPYSL2 expression in the pathway of receptor-mediated axon growth repulsion (Figure 1).

Koide et al. investigated 20 tag single nucleotide polymorphisms (SNPs) of DPYSL2 in 1464 patients with schizophrenia and 1310 healthy control subjects. They detected positive associations between two SNPs (rs2585458 and rs4733048) and schizophrenia in a screening population of 384 cases and 384 controls. However, they could not replicate these potential associations in a confirmatory population of 1080 cases and 926 controls. They also conducted statistical calculations of genotypic prediction of 96 un-genotyped variants using the MACH program. None of the variations was associated with schizophrenia in the current study. The authors concluded that DPYSL2 might not be involved in susceptibility to schizophrenia in the Japanese population. They also faced the difficulty of psychiatric genetics.

Two etiological scenarios are possible for genetically complex and common diseases like schizophrenia: the 'common variant common disease' model, which Koide et al. used, and the 'multiple rare variants common disease' model. Several current studies highlight the validity of the latter approach and of deep analyses (rare variations and copy number of variants) of a target gene in studies of psychiatric diseases. The strategy to identify multiple rare mutations of large effects leads to clarifying the etiology of schizophrenia as a prototype of clinical manifestations. Unfavorable gene-environment interactions have long been involved in the etiology of schizophrenia, but the biological mechanisms are not yet completely understood. 


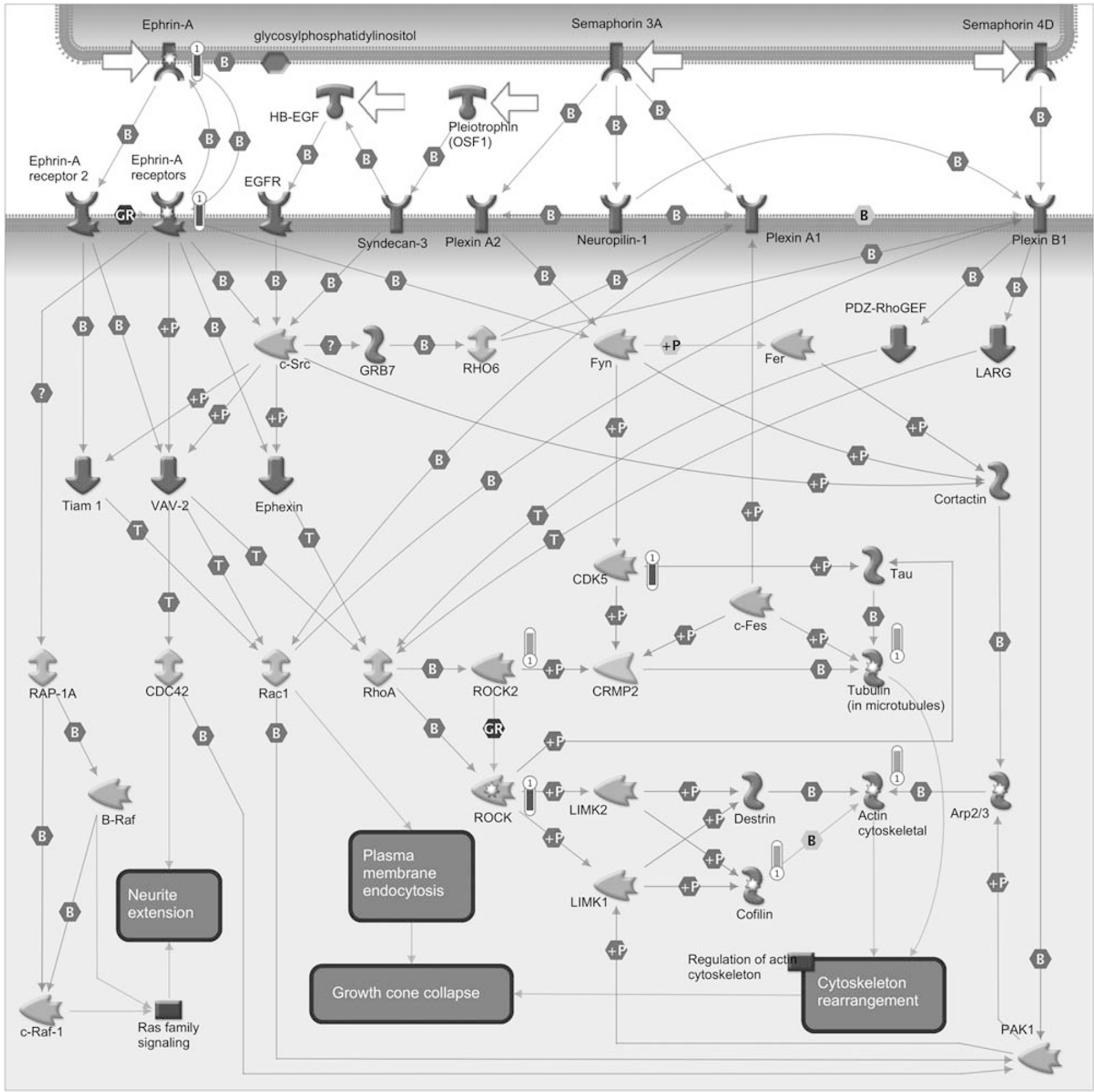

Figure 1 The pathway of receptor-mediated axon growth repulsion. Several molecules have been shown to phosphorylate CRMP2 (DPYLS2), and CRMP2 binds to tubulin heterodimers to promote microtubule assembly, which is important for axon outgrowth and branching. The red and blue bars show the gene expression levels using our unpublished microarray data in lymphoblastoid cells derived from schizophrenic patients and control subjects. When the gene expressions in patients are lower than in control subjects, the level is represented by a blue bar. A red bar shows increased gene expression of molecules in schizophrenia compared with controls. The MetaCore platform (http://www.infocom.co.jp/bio/develop/metacore.html) was used for all data calculations. A full color version of this figure is available at the Journal of Human Genetics journal online.

Schizophrenic patients have multiple symptoms, and, therefore, the problem of disease heterogeneity is always involved in any research sample. As for many common diseases, because the homeostasis between networks of molecular cascades fails, an abnormal condition occurs. Therefore, in further work, we should focus on combinations of profiling of metabolomics, genomics and clinical manifestations of a subgroup of patients with schizophrenia. That could be possible resolution for the hard road in psychiatric genetics.

1 Pei, Y. F., Li, J., Zhang, L., Papasian, C. J. \& Deng, H. W. Analyses and comparison of accuracy of different genotype imputation methods. PLoS One 3, e3551 (2008).
2 Goshima, Y., Nakamura, F., Strittmatter, P. \& Strittmatter, S. M. Collapsin-induced growth cone collapse mediated by an intracellular protein related to UNC-33. Nature 376, 509-514 (1995).

3 Uchida, Y., Ohshima, T., Sasaki, Y., Suzuki, H., Yanai, S. Yamashita, N. et al. Semaphorin3A signalling is mediated via sequential Cdk5 and GSK3beta phosphorylation of CRMP2: implication of common phosphorylating mechanism underlying axon guidance and Alzheimer's disease. Genes Cells 10, 165-179 (2005). 4 Arimura, N., Inagaki, N., Chihara, K., Ménager, C., Nakamura, N., Amano, M. et al. Phosphorylation of collapsin response mediator protein-2 by Rho-kinase. 
Evidence for two separate signaling pathways for growth cone collapse. J. Biol. Chem. 275, 23973-23980 (2000).

5 Fukata, Y., Itoh, T. J., Kimura, T., Ménager, C., Nishimura, T., Shiromizu, T. et al. CRMP-2 binds to tubulin heterodimers to promote microtubule assembly. Nat. Cell Biol. 4, 583-591 (2002).

6 Kawano, Y., Yoshimura, T., Tsuboi, D., Kawabata, S., Kaneko-Kawano, T., Shirataki, H. et al. CRMP-2 is involved in kinesin-1-dependent transport of the Sra-1/WAVE1 complex and axon formation. Mol. Cell Biol. 25, 9920-9935 (2005).
7 Inagaki, N., Chihara, K., Arimura, N., Ménager, C., Kawano, Y., Matsuo, N. et al. CRMP-2 induces axons in cultured hippocampal neurons. Nat. Neurosci. 4, 781-782 (2001).

8 Martins-de-Souza, D., Maccarrone, G., Wobrock, T., Zerr, I., Gormanns, P., Reckow, S. et al. Proteome analysis of the thalamus and cerebrospinal fluid reveals glycolysis dysfunction and potential biomarkers candidates for schizophrenia. J. Psychiatr. Res. (e-pub ahead of print 12 May 2010)

9 Nakata, K., Ujike, H., Sakai, A., Takaki, M., Imamura, T., Tanaka, Y. et al. The human dihydropyrimidinaserelated protein 2 gene on chromosome $8 \mathrm{p} 21$ is asso- ciated with paranoid-type schizophrenia. Biol. Psychiatry 53, 571-576 (2003).

10 Zhao, X., Tang, R., Xiao, Z, Shi, Y., Feng G., Gu, N. et al. An investigation of the dihydropyrimidinase-like 2 (DPYSL2) gene in schizophrenia: genetic association study and expression analysis. Int. J. Neuropsychopharmacol. 9, 705-712 (2006).

11 Koide, T., Aleksic, B., Ito, Y., Usui, H., Yoshimi, A., Inada, T. et al. A two-stage case-control association study of the dihydropyrimidinase-like 2 gene (DPYSL2) with schizophrenia in Japanese subjects. J. Hum. Genet. 55, 469-472 (2010). 